The Impact of Large-Scale Surveys on Pulsating Star Research ASP Conference Series, Vol. 209, 2000

L. Szabados \&; D. W. Kurtz, eds.

\title{
Surveys and the Discovery of New Variable White Dwarfs
}

\author{
G. Vauclair ${ }^{1}$, N. Dolez ${ }^{1}$, Fu Jian Ning ${ }^{1}$, M. Chevreton ${ }^{2}$, D. Homeier ${ }^{3}$, \\ D. Koester ${ }^{3}$
}

Abstract. We report the discovery of a new variable DA white dwarf (ZZ Ceti): PG1541+650, identified as a potential ZZ Ceti candidate in the Hamburg Quasar Survey.

Surveys have recently been undertaken whose aim is to search for new quasars and other interesting blue objects. As a by-product, such surveys have led to the discovery of a large number of white dwarfs and hot subdwarfs. In addition to the Palomar-Green survey (Green, Schmidt, \& Liebert 1986), the Kiso survey (Noguchi, Maehara, \& Kondo 1980; Kondo, Noguchi, \& Maehara 1984), the Edinburgh-Cape survey (EC: Stobie et al. 1988, 1992), the Hamburg quasar survey (HQS: Hagen et al. 1995), among others, are very productive in terms of the discovery of new white dwarfs. Among the newly discovered white dwarfs, some have atmospheric parameters which fit with those of the three known instability strips for the pulsating PG1159, DB and DA white dwarfs. They are currently tested for photometric variability and some of them have been confirmed as new pulsating white dwarfs. Considering the rich potential of the asteroseismology of the late stages of stellar evolution for our understanding of stellar structure and evolution, increasing the number of known pulsating white dwarfs whose asteroseismological study may be undertaken is an important issue. At present, in addition to 10 pulsating Planetary Nebula Nuclei (PNNV), only 5 pulsating pre-white dwarfs of PG1159 type, 8 pulsating DB white dwarfs (DBV) and 28 pulsating DA white dwarfs (DAV or ZZ Ceti stars) are known.

Among the most recently discovered pulsating white dwarfs from these surveys, one finds: HS2324+3944 (Silvotti 1996; Dreizler et al. 1996) from the HQS and RXJ2117+3412 (Vauclair et al. 1993) from the ROSAT X-ray survey, in the PG1159 instability strip; KUV05134+2605 (Grauer, Wegner, \& Liebert 1989) from the KISO survey and EC20058-5234 (O'Donoghue 1995; Koen et al. 1995) from the EC survey, in the DB instability strip; HS0507+0435 (Jordan et al. 1998) from the HQS, KUV08368+4026 and KUV11370+4222 (Vauclair et al. 1996) from the KISO survey, EC14012-1446 (Stobie et al. 1995) and EC23487-2424 (Stobie et al. 1993) from the EC survey, in the DA instability strip.

Homeier et al. (1998) have analysed a set of DA white dwarfs from the HQS. A sub-sample of these have atmospheric parameters $\left(T_{\text {eff }}\right.$ and $\left.\log g\right)$ in the

\footnotetext{
${ }^{1}$ Laboratoire d'Astrophysique, Observatoire Midi-Pyrénées, 14 avenue Édouard Belin, F-31400 Toulouse, France

${ }^{2}$ Observatoire de Paris-Meudon, Place J. Janssen, 92195 Meudon, France

${ }^{3}$ Institut für Theoretische Physik und Astrophysik, Leibnizstr. 15, Universität Kiel, D-24098 Kiel, Germany
} 
range of the $\mathrm{ZZ}$ Ceti instability strip. We have undertaken to observe them to search for new ZZ Ceti variables. We report here on the discovery of the new ZZ Ceti star PG1541+650. PG1541+650 was already identified as a DA white dwarf in the PG survey. It was again identified in the HQS, and the subsequent atmospheric analysis of Homeier et al. (1998) indicates an effective temperature of $12,000 \mathrm{~K}$, right in the ZZ Ceti instability strip. PG1541+650 was observed with the Chevreton 3-channel photometer at the 2-m telescope of the Pic du Midi observatory on August 23, 1998. It was discovered to be variable with a period of about $11 \mathrm{~min}$. We re-observed PG1541+650 at the 1.93-m telescope of the Haute Provence observatory on 14 and 15 May 1999. The Fourier Transform of the light curves is dominated by a $1.457-\mathrm{mHz}$ frequency (686-s period) with an amplitude of $0.045 \mathrm{mag}$. A second frequency at $1.780 \mathrm{mHz}$ (562-s period) is also present with a smaller amplitude of $0.015 \mathrm{mag}$. The first harmonic of the dominant frequency is seen at $2.912 \mathrm{mHz}$ with an amplitude of $0.012 \mathrm{mag}$.

The continuing search for new variable white dwarfs in the samples issued from recent surveys is encouraging. With the discovery reported here, the number of known ZZ Ceti white dwarfs is now 29, among which 6 have been found from such surveys.

\section{References}

Dreizler, S., Werner, K., Heber, U, \& Engels, D. 1996, A\&A, 309, 820

Grauer, A., Wegner, G., \& Liebert, J. 1989, AJ, 98, 221

Green, R., Schmidt, M., \& Liebert, J. 1986, ApJS, 61, 305

Hagen, H.-J., Groote, D., Engels, D., \& Reimers, D. 1995, A\&AS, 111, 195

Homeier, D., Koester, D., Hagen, H.-J., et al. 1998, A\&A, 338, 563

Jordan, S., Koester, D., Vauclair, G., et al. 1998, A\&A, 330, 277

Koen, C., O'Donoghue, D., Stobie, R. S., Kilkenny, D., \& Ashley, R. 1995, MNRAS, 277, 913

Kondo, M., Noguchi, T., \& Maehara, H. 1984, Ann. Tokyo Astron. Obs. 20, 130

Noguchi, T., Maehara, H., \& Kondo, M. 1980, Ann. Tokyo Astron. Obs. 18, 55

O'Donoghue, D. 1995, in Proc. 9th European Workshop on white dwarfs stars, ed. D. Koester \& K. Werner (Berlin: Springer), 297

Silvotti, R. 1996, A\&A, 309, L23

Stobie, R. S., Morgan, D. H., Bhatia, R. K., et al. 1988, in IAU Coll. 95, The Second Conference on Faint Blue Stars, ed. A. G. Davis Philip, D. S. Hayes, \& J. W. Liebert (Schenectady: L. Davis Press), 493

Stobie, R. S., Chen, A., O'Donoghue, D., \& Kilkenny, D. 1992, in ASP Conf. Ser. Vol. 30, Variable Stars and Galaxies, ed. B. Warner (San Francisco: ASP), 87

Stobie, R. S., Chen, A., O'Donoghue, D., \& Kilkenny, D. 1993, MNRAS, 263, L13

Stobie, R. S., O'Donoghue, D., Ashley, R., et al. 1995, MNRAS, 272, L21

Vauclair, G., Belmonte, J. A., Pfeiffer, B., et al. 1993, A\&A, 267, L35

Vauclair, G., Dolez, N., Fu Jian Ning, \& Chevreton, M. 1996, A\&A, 322, 155 